\title{
Lesión miocárdica en una paciente con lupus eritematoso sistémico: Un reto diagnóstico
}

\author{
Myocardial lesion in a patient with systemic lupus erythematosus: A diagnostic challenge
}

\author{
Milly J. Vecino-Moreno, ${ }^{1,2}$, Mario A. Bautista-Vargas ${ }^{1,2}$, Ángela M. Herrera-Peña ${ }^{3}$, \\ Fernando A. Guerrero-Pinedo 4 y David A. Aguirre-Valencia ${ }^{1,5 *}$
}

${ }^{1}$ Departamento de Reumatología, Facultad de Medicina, Universidad Icesi; ${ }^{2}$ GIRAT: Grupo de Investigación en Reumatología, Autoinmunidad y Medicina Traslacional, Fundación Valle del Lili y Universidad Icesi; ${ }^{3}$ Departamento de Cardiología, Facultad de Medicina, Universidad Icesi; ${ }^{4}$ Departamento de Cardiología Intervencionista, Fundación Valle del Lili; ${ }^{5}$ Departamento de Reumatología, Fundación Valle del Lili. Cali, Colombia

\section{Resumen}

Mujer de 37 años con antecedentes de lupus eritematoso sistémico (LES), tromboembolia pulmonar y trombosis venosa profunda, quien consultó por dolor torácico, con hallazgos de elevación de la troponina, disfunción sistólica del ventrículo izquierdo y alteraciones de la contractilidad segmentaria, además de anti-DNA elevado y complemento consumido. La angiografía coronaria mostró estenosis grave en el tercio medio de la arteria descendente anterior, que fue intervenida con angioplastia más stent medicado. La resonancia magnética cardiaca reveló infarto agudo de miocardio extenso en el territorio de la arteria descendente anterior, sin miocarditis. Se descartó compromiso cardiaco por LES, se continuó igual terapia inmunosupresora, se añadió manejo para enfermedad coronaria y egresó por buena evolución. El enfoque de las causas de lesión miocárdica en un paciente con LES supone un reto, dadas las múltiples posibilidades en el espectro de compromiso cardiaco, ya que varias estructuras se pueden ver afectadas. La miocarditis y la pericarditis se consideran las más frecuentes, pero el compromiso coronario no puede descartarse, independientemente de la edad y de la presencia de factores de riesgo tradicionales, puesto que el componente inflamatorio de la patología autoinmunitaria confiere un desarrollo acelerado de ateroesclerosis. La multimodalidad diagnóstica y el enfoque interdisciplinario son necesarios para aclarar el mecanismo de la lesión y así brindar un tratamiento dirigido.

Palabras clave: Lupus eritematoso sistémico. Compromiso cardiaco. Diagnóstico.

\section{Abstract}

A 37-year-old woman with a history of systemic lupus erythematosus (SLE), pulmonary thromboembolism and deep vein thrombosis consulted for chest pain, with findings of elevated troponin, left ventricular systolic dysfunction, and segmental contractility abnormalities, as well as elevated anti-DNA and complement consumption. Coronary angiography showed severe stenosis of the middle third of the anterior descending artery, which was treated with angioplasty plus a medicated stent. Cardiac magnetic resonance imaging revealed an extensive acute myocardial infarction in the area supplied by the anterior descending artery, without myocarditis. Cardiac involvement in SLE was ruled out, immunosuppressive treatment was

\section{Correspondencia:}

*David A. Aguirre-Valencia E-mail: david.aguirre@fvl.org.co
Fecha de recepción: 10-08-2020

Fecha de aceptación: 06-11-2020 DOI: 10.24875/RCCAR.M21000105
Disponible en internet: 07-01-2022 Rev Colomb Cardiol. 2021;28(6):634-638 www.rccardiologia.com 0120-5633 / @ 2021 Sociedad Colombiana de Cardiología y Cirugía Cardiovascular. Publicado por Permanyer. Este es un artículo open access bajo la licencia CC BY-NC-ND (http://creativecommons.org/licenses/by-nc-nd/4.0/). 
maintained, coronary disease treatment was added, and she was discharged due to improvement. Identifying the causes of myocardial insult in a patient with SLE is challenging, given the multiple possibilities across the cardiac involvement spectrum, in which several structures may be affected. Myocarditis and pericarditis are considered to be the most frequent, but coronary involvement cannot be ruled out, regardless of age or the presence of traditional risk factors, since the inflammatory component of the autoimmune disease accelerates the development of atherosclerosis. Multimodal diagnostics and an interdisciplinary approach are necessary to clarify the mechanism of injury and provide targeted treatment.

Key words: Systemic lupus erythematosus. Cardiac involvement. Diagnosis.

\section{Introducción}

El lupus eritematoso sistémico (LES) es una enfermedad autoinmunitaria que afecta predominantemente a mujeres en edad fértil, presentando un gran compromiso sistémico, y por tanto sus síntomas se relacionarán con los órganos comprometidos. El dolor torácico es un síntoma frecuente en este grupo de pacientes (hasta un $15 \%$ de ellos ingresan a urgencias por este motivo) y se convierte en un reto diagnóstico, porque dentro del enfoque se deben incluir causas de origen pulmonar, gastrointestinal, musculoesquelético y cardiaco. Este último incluye alteraciones miocárdicas, valvulares y pericárdicas, sin olvidar la enfermedad coronaria'.

\section{Caso clínico}

Mujer de 37 años con antecedente de lupus eritematoso sistémico quien debutó 20 años atrás con nefritis lúpica de clase III, recibiendo manejo con azatioprina, cloroquina y prednisona. Durante el curso de su enfermedad desarrolla un episodio de tromboembolia pulmonar y se documenta triple positividad de anticuerpos antifosfolípidos, diagnosticando así un síndrome antifosfolípido (SAF) secundario, por lo cual se adicionó manejo con warfarina de forma indefinida. Tras 12 años de enfermedad presenta una recaída de la nefritis lúpica, se realiza nueva biopsia y se encuentra nefritis lúpica de clase $\mathrm{V}$; sin embargo, la paciente rehúsa un cambio de inmunosupresión y decide continuar con igual manejo. Debido a la persistencia de proteinuria en rango nefrótico y el edema de miembros inferiores, acepta la modificación del esquema de azatioprina por micofenolato mofetilo. La evolución clínica es hacia la mejoría tras el ajuste del manejo inmunosupresor.

Consulta al servicio de urgencias por un cuadro de 24 horas de evolución que inició con dolor torácico súbito, opresivo, asociado a disnea de pequeños esfuerzos que progresó hasta el reposo. Fue evaluada inicialmente en una primera institución donde se le realizó un electrocardiograma que mostró poca progresión de la onda R de V1-V4, imagen de $Q$ en V1V3 y elevación del segmento ST de V1-V3 y en AVL. Recibió manejo con doble antiagregación plaquetaria. Se documentaron creatina fosfocinasa (CPK) total y troponina elevadas, sin curva; inicialmente se consideró una baja probabilidad de síndrome coronario agudo y se sospechó miocarditis lúpica, por lo que se inició manejo con pulsos de metilprednisolona, pero la evolución fue tórpida, persistiendo con dolor torácico, y por ello se realizó un ecocardiograma transtorácico que evidenció una fracción de eyección del ventrículo izquierdo del $28 \%$, trastornos segmentarios de la contractilidad (acinesia del septo anterior, pared anterior en segmento apical, medio y parte basal), ventrículo derecho no dilatado con función sistólica preservada, aurícula izquierda levemente dilatada, válvulas sin alteraciones significativas, sin signos de hipertensión pulmonar y mínimo derrame pericárdico posterior. Posteriormente fue remitida a una institución de cuarto nivel, donde su caso se reevaluó, con medición de troponina I ultrasensible con un valor inicial de $22,947.5 \mathrm{ng} / \mathrm{l}$ y un control a las 2 horas de 23,063.8 ng/l. La CPK estaba ligeramnente aumentada en $182 \mathrm{U} / \mathrm{l}$. El perfil autoinmunitario fue anti-DNA positivo por enzimoinmunoanálisis (EIA) con $135.5 \mathrm{IU} / \mathrm{ml}$, pero negativo por inmunofluorescencia indirecta (IFI), C3 bajo en 80.11 (VR: $90-180 \mathrm{mg} / \mathrm{dl}$ ) y $\mathrm{C} 4$ bajo en 6.32 (VR: 10-40 mg/dl). Se tomó la decisión de realizar una arteriografía coronaria, en la que se encontró en la arteria descendente anterior una estenosis del $95 \%$ en el segmento medio, con un vaso distal levemente adelgazado y flujo distal TIMI (Thrombolysis in Miocardial Infarction) 1. Se realizaron angioplastia y colocación de stent medicado, con buen resultado angiográfico, TIMI 3 (Fig. 1). Por la alteración del perfil inmunológico con anti-DNA por técnica ELISA (Enzyme-Linked Immunosorbent Assay) francamente elevado y complemento sérico levemente consumido, sumado a la presencia de derrame pericárdico, persistía la duda sobre si existía 


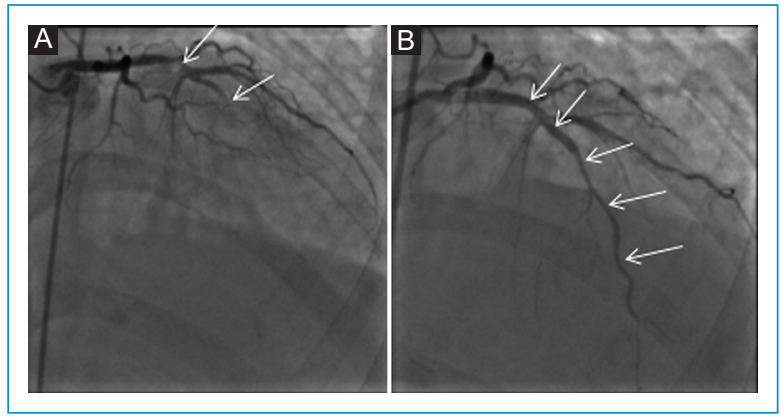

Figura 1. A: Estenosis grave de la descendente anterior media con flujo distal TIMI 1. B: Angioplastia más implante de stent medicado en la descendente anterior, flujo distal TIMI 3.

compromiso miocárdico adicional por actividad lúpica (SLEDAI-2K:10), por lo que se realizó una resonancia magnética cardiaca que reportó un infarto agudo de miocardio extenso en el territorio de la arteria descendente anterior, con compromiso de la pared anterior y del septo interventricular en los planos medio apical y del ápex ventricular, con signos de obstrucción microvascular (Fig. 2), dilatación del ventrículo izquierdo y deterioro moderado de la función sistólica, volúmenes ventriculares derechos y función sistólica dentro de la normalidad, derrame pericárdico moderado, sin signos de miocarditis (Fig. 3). Tuvo una buena evolución clínica intrahospitalaria tras el ajuste del manejo médico y continuó con igual esquema de inmunosupresión, por lo que fue dada de alta 8 días después de su ingreso.

\section{Discusión}

En los pacientes con LES, el corazón puede verse afectado en el curso de la enfermedad hasta en un $50 \%$ de los $\operatorname{casos}^{2}$. La pericarditis es la manifestación cardiaca más común en el LES. Aproximadamente el $25 \%$ de los pacientes desarrollan pericarditis sintomática durante el curso de la enfermedad, con mayor frecuencia junto a pleuritis asociada. Por otra parte, la miocarditis por LES es rara ${ }^{3}$.

La enfermedad coronaria que se manifiesta como infarto agudo de miocardio es hasta nueve veces más frecuente en los pacientes con LES que en la población general, llegando a ser hasta 50 veces mayor en las mujeres con LES en edades entre los 35 y 44 años comparadas con población sana del mismo sexo y la misma edad ${ }^{4}$. De igual forma se debe tener en cuenta que, en los pacientes con LES y SAF, hasta el $75 \%$ de los episodios de infarto agudo de miocardio tienen

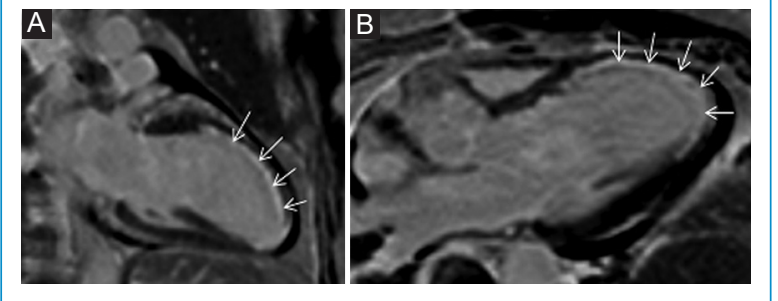

Figura 2. A: Eje largo de dos cámaras, contraste tardío con infarto extenso en el territorio de la descendente anterior. B: Eje largo de tres cámaras, contraste tardío con infarto extenso en el territorio de la descendente anterior.

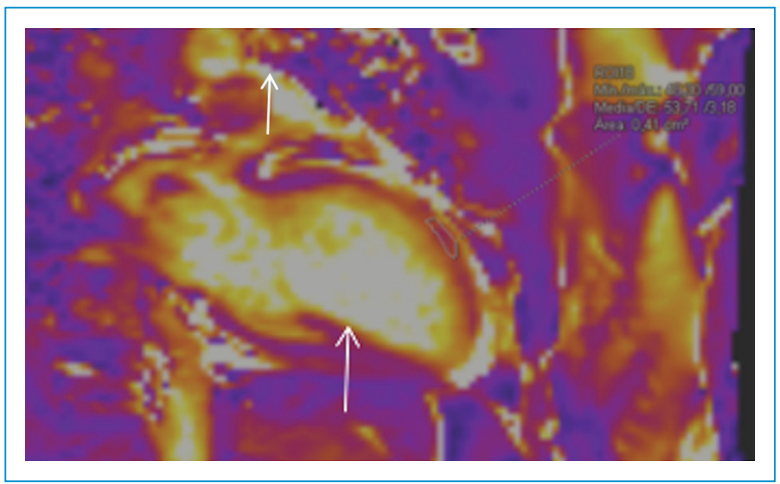

Figura 3. Mapa de color de T2 que muestra edema en el territorio de la descendente anterior (área demarcada), que contrasta con el color normal en la cara posterior (flechas).

coronarias sanas, mientras que solo en un $25 \%$ se evidencia estenosis significativa ${ }^{5}$; esto se relaciona directamente con el tiempo de evolución de la enfermedad (LES y SAF), ya que se ha encontrado que con menor tiempo de evolución de la enfermedad los eventos coronarios se relacionan principalmente con arteritis coronaria, miocarditis, trombosis coronaria 0 embolización con recanalización espontanea ${ }^{6}$, mientras que en aquellos pacientes con mayor tiempo de evolución de la enfermedad se observa un desarrollo acelerado de ateroesclerosis coronaria, relacionada con valores elevados de colesterol y triglicéridos, y valores más bajos de lipoproteínas de alta densidad, comparados con la población general de la misma edad y el mismo sexo².

La ateroesclerosis es considerada una enfermedad inflamatoria y autoinmunitaria. Es así que mientras el desarrollo de anticuerpos específicos contra antígenos, como las proteínas de choque térmico HSP60/65 y la 
B2-glucoproteína-1, promueven una rápida progresión de la ateroesclerosis por medio de la lisis de las células endoteliales, otros factores, como los anticuerpos contra las lipoproteínas de baja densidad oxidadas (oxLDL), impiden la fagocitosis de estas oxLDL por los macrófagos, comportándose como factores protectores, ya que disminuyen la progresión de la placa ateroesclerótica ${ }^{7}$.

Otros factores, como el síndrome nefrótico y la proteinuria, están asociados una dislipidemia grave y un aumento del riesgo protrombótico. Por su parte, la activación del complemento también contribuye a la formación de la placa ateroesclerótica, estimulando la activación de células endoteliales y el reclutamiento de linfocitos hacia los sitios de inflamación, inhibiendo enzimas como la 27-hidrolasa que se encargan de facilitar el transporte del colesterol fuera de la pared arterial y estimulando las moléculas de adhesión a la pared vascular, aumentando así el reclutamiento de monocitos hacia estos lugares. El uso crónico de esteroides, si bien ha sido planteado como un factor de riesgo para la aterosclerosis debido a su potencial de desarreglo metabólico como causa de elevación de la presión arterial, el colesterol total y la glucosa, y el cambio en la distribución de la grasa corporal, pudiera funcionar como un factor protector, inicialmente, en casos con cuadros inflamatorios graves y sostenidos, con manifestaciones clínicas moderadas a graves, gracias a su potente efecto antiinflamatorio ${ }^{8}$.

En el caso aquí presentado se tuvo la duda sobre si el compromiso cardiaco, evidente por la elevación de biomarcadores, la disfunción sistólica ventricular izquierda y la alteración de la contractilidad segmentaria, era debido a una miocarditis por enfermedad lúpica o a un evento coronario agudo; esta útima etiología fue tenida en cuenta porque la paciente presentaba un estado de hipercoagulación no solo debido al LES de base, sino también a la triple positividad de anticuerpos antifosfolípidos ${ }^{5}$ y además en ella se veía reflejada la paradoja de la inflamación, puesto que se vio expuesta a dosis de glucocorticoides durante muchos años, medicamentos conocidos por su efecto nocivo sobre el sistema cardiovascular ${ }^{9}$, pero de otro lado, a pesar de dicho manejo se mantuvo activa, con recaídas (episodios de nefritis lúpica), para las que rehusó un cambio de manejo inmunomodulador, y todo lo anterior imposibilitaba descartar el daño endotelial y la trombosis miocárdica como causa de este evento.

Los marcadores de autoinmunidad, anti-DNA elevado y complemento consumido, apoyaban la posibilidad de actividad lúpica; sin embargo, en el seguimiento la paciente tenía valores bajos de complemento de forma crónica y el anti-DNA solo fue positivo por EIA, no por IFI. Esto fue corroborado una vez se realizó la resonancia magnética cardiaca, en la que no se evidenciaron alteraciones en los mapas de T2 que sugirieran miocarditis y en su lugar se vieron las alteraciones en el territorio correspondiente a la descendente anterior, de manera que el abordaje inicial con coronariografía y el manejo previo con carga antiisquémica fueron adecuados. Dado que cursaba con enfermedad inflamatoria sistémica de larga data, con uso crónico de esteroides y manifestaciones graves de la enfermedad, con factores protrombóticos adicionales, como la coexistencia de SAF, la posibilidad pretest de evento coronario agudo, aun con edad joven y en ausencia de factores de riesgo tradicionales, no debería considerarse baja.

La mortalidad asociada a infarto agudo de miocardio en los pacientes con LES y SAF es también mayor que en la población general, siendo calculada en un 13.9\% en grandes cohortes como el Euro-Phospholipid Project ${ }^{10}$, tal vez relacionado con la menor sospecha diagnóstica que lleva a la instauración del tratamiento de forma más tardía.

Un aspecto importante en la mayoría de estos pacientes con LES y SAF que se presentan con dolor torácico, al ser un factor de confusión como ocurrió en nuestra paciente, es la diferenciación entre actividad lúpica y causas como tromboembolia pulmonar o infarto agudo de miocardio.

La multimodalidad en la evaluación de estos pacientes, aun en el evento agudo, permite aclarar el escenario. Los exámenes de laboratorio y diferentes técnicas de imagen se deben conjugar con el criterio clínico multidisciplinario para la toma de decisiones. Dentro de ellas destaca la angiotomografía coronaria para la detección de placas no calcificadas de baja atenuación, las cuales son características de los vasos en placas coronarias inestables, cambios directamente implicados en la muerte tardía en el LES ${ }^{11}$; anexo a ello, el grosor de la íntima-media carotídea, evaluado mediante ecografía en modo B o M, es una de las medidas no invasivas para valorar y seguir la aterosclerosis subclínica, según lo recomendado por la American Heart Association. Un metaanálisis reciente en pacientes con LES mostró que la tasa de aterosclerosis aumentó en comparación con los controles sanos emparejados por edad y sexo ${ }^{12}$.

La resonancia magnética cardiaca es una herramienta muy valiosa en la cardiología moderna, y en esta población especial su uso ya ha sido evaluado, 
con resultados positivos en pequeños grupos de pacientes en que se ha visto que hasta el $43 \%$ tienen hallazgos anormales en la resonancia magnética cardiaca, y los patrones de distribución y realce del gadolinio permiten diferenciar mecanismos de lesión isquémicos y no isquémicos; además, es útil en la detección de compromiso subclínico e incluso permite la identificación de otros posibles factores de riesgo cardiovascular $^{13}$.

\section{Conclusiones}

El espectro del compromiso cardiaco por LES es amplio, más allá del compromiso miocárdico o pericárdico, y la ocurrencia del síndrome coronario agudo no solo es factible, sino que es frecuente, por lo que en pacientes con patología autoinmunitaria se debería mantener un alto nivel de sospecha frente a esta posibilidad y actuar en consecuencia. La evaluación multidisciplinaria y el uso de diversas herramientas diagnósticas, tanto de laboratorio como de imagen, son de gran ayuda.

\section{Financiamiento}

Los autores declaran que no recibieron financiamiento de parte de ninguna institución.

\section{Conflicto de intereses}

Los autores declaran no tener conflictos de intereses.

\section{Responsabilidades éticas}

Protección de personas y animales. Los autores declaran que para esta investigación no se han realizado experimentos en seres humanos ni en animales.
Confidencialidad de los datos. Los autores declaran que han seguido los protocolos de su centro de trabajo sobre la publicación de datos de pacientes.

Derecho a la privacidad y consentimiento informado. Los autores han obtenido el consentimiento informado de los pacientes y/o sujetos referidos en el artículo. Este documento obra en poder del autor de correspondencia.

\section{Bibliografía}

1. Modi M, Ishimori ML, Sandhu VK, Wallace DJ, Weisman MH. Chest pain in lupus patients: the emergency department experience. Clin Rheumatol. 2015;34:1969-73.

2. Kreps A, Paltoo K, McFarlane I. Cardiac manifestations in systemic lupus erythematosus: a case report and review of the literature. Am J Med Case Rep. 2018;6:180-3.

3. Miner JJ, Kim AHJ. Cardiac manifestations of systemic lupus erythematosus. Rheum Dis Clin North Am. 2014;40:51-60.

4. Manzi S, Meilahn EN, Rairie JE, Conte CG, Medsger TA Jr, Jansen-McWilliams L, et al. Age-specific incidence rates of myocardial infarction and angina in women with systemic lupus erythematosus: comparison with the Framingham Study. Am J Epidemiol. 1997; $145: 408-15$

5. Nazir S, Tachamo N, Lohani S, Hingorani R, Poudel DR, Donato A. Acute myocardial infarction and antiphospholipid antibody syndrome: a systematic review. Coron Artery Dis. 2017;28:332-5.

6. Farooq A, Ullah A, Ali F, Yasin H, Amjad W, Pervaiz M. Acute myocardial infarction in young systemic lupus erythematosus patient with normal coronary arteries. Cureus. 2017;9:e1370.

7. Blasi $\mathrm{C}$. The autoimmune origin of atherosclerosis. Atherosclerosis. 2008;201:17-32

8. Sherer $\mathrm{Y}$, Zinger $\mathrm{H}$, Shoenfeld $\mathrm{Y}$. Atherosclerosis in systemic lupus erythematosus. Autoimmunity. 2010;43:98-102.

9. Souverein PC, Berard A, Van Staa TP, Cooper C, Egberts AC, Leufkens HG, et al. Use of oral glucocorticoids and risk of cardiovascular and cerebrovascular disease in a population based case-control study. Heart. 2004;90:859-65

10. Cervera R, Serrano R, Pons-Estel GJ, Ceberio-Hualde L, Shoenfeld Y, de Ramón $\mathrm{E}$, et al. Morbidity and mortality in the antiphospholipid syndrome during a 10-year period: a multicentre prospective study of 1000 patients. Ann Rheum Dis. 2015;74:1011-8.

11. Stojan G, Li J, Budoff M, Arbab-Zadeh A, Petri MA. High-risk coronary plaque in SLE: low-attenuation non-calcified coronary plaque and positive remodelling index. Lupus Sci Med. 2020;7:e000409.

12. Wu G-C, Liu H-R, Leng R-X, Li XP, Li XM, Pan HF, et al. Subclinical atherosclerosis in patients with systemic lupus erythematosus: a systemic review and meta-analysis. Autoimmun Rev. 2016;15:22-37.

13. Burkard T, Trendelenburg M, Daikeler T, Hess C, Bremerich J, Haaf P, et al. The heart in systemic lupus erythematosus - a comprehensive approach by cardiovascular magnetic resonance tomography. PLoS One. 2018;13:e0202105. 